\title{
Natural Vs. Synthetic Food: Which Is Better?
}

\author{
A Wangsa ${ }^{1}$, H. Hery ${ }^{2}$ J T Purba ${ }^{3}$ \\ \{andrean.wangsa@stud.iuli.ac.id ${ }^{1}$, hery.fik@uph.edu ${ }^{2}$, john.purba@uph.edu ${ }^{3}$ \} \\ ${ }^{1}$ International University Liaisons Indonesia, Indonesia \\ ${ }^{2,3}$ UniversitasPelita Harapan, Indonesia
}

\begin{abstract}
In this food chemistry research, we researched on food additive regulations based on the US\& UK, then discussed the controversial topic of whether synthetic(artificial) foods \& food additives are better or worse than those that are of natural sources. The purpose of this research is to educate ourselves and other people regarding that matter and to eventually reduce the number of food-chemophobic people as food industries use those ingredients to benefit consumers in economic, sensory, or nutritional perspectives. No synthetic food will have the same organoleptic properties as the real food; however nutritional values can be mimicked or even better due to the controllable quality and increased stability.
\end{abstract}

Keywords: Food, chemistry, nutrition, additives

\section{Introduction}

When we think of natural vs. synthetic, we often think that natural is always better because we are brainwashed by "the naturalistic fallacy" to think that synthetic items are dangerous and the "fake version" of natural foods that will never be $100 \%$ same as the natural food. It is true that synthetic items will never be the same as natural ones, however, the truth is that synthetic isn't always bad, and sometimes it may even be better for people to consume because it is more customizable. Little did we know that most of the food we eat contain synthetic compounds. An example of how synthetic compounds are beneficial would be the invention of synthetic drugs to counter against naturally occurring toxins. Every molecule has to be analyzed by a case by case attitude instead of thinking that all molecules are created equally or having the mindset to think that natural is always better than synthetic. A part of why artificial compounds have a bad reputation is due to them being used in most processed foods, which are generally high in fats and simple sugars, but low in fiber. However, the processing is responsible for this, not the additives. In the food industry, a lot of processing takes place "from grass to glass" to improve the sanitation, shelf life, and other physical/chemical quality of the food. But lots of its naturally occurring nutrients, pigments, aromas, or flavor compounds are lost during processing, this is the reason behind why food chemists add synthetic food additives.

\section{Discussion}

As of now according to the USFDA(United States Food and Drug Administration), the term "natural" can be used when the substance is derived from plants, animals, or fermentation, ex: "natural flavoring" means that it is derived from either those 3 , whereas artificial additives refer to the additive being made in a lab from scratch[1]. However, people should also realize that the "natural" compound may also be processed and have some synthetic additives before it is put in the food product, ex: the addition of n-hexane in the 
production of margarine (this is not a problem as hexane is only used as a solvent and has a relatively low boiling point (and its other ideal non-polar solvent properties) which means most/all of it has already evaporated when the product is finished)[2].

Natural is not always better because not everything from nature is safe/good, keep in mind that rattlesnake venom, arsenic in wild almonds, and molds are also natural. I think that people should not have the fear of synthetic materials, or chemicals (chemophobia) because everything we eat and everything around us, in fact, we ourselves are actually made of chemicals[3]. Also, they should realize that the addition of these substances is to profit the customer, from either a safety, sensory, economic, or nutritional perspective[4]. Synthetic chemicals may be beneficial for people because they are cheaper to synthesize, which means that the price can be more affordable for everyone because some natural items are relatively rare/expensive.

An example would be vanillin which is naturally derived from vanilla beans [5], nowadays is synthesized in the lab by synthetic chemists through organic synthesis methods using petroleum derivatives which is the most sufficient method to use in comparison to the extraction of pure vanilla beans, or another lignin bioconversion method that includes microbes[6], which is great because it is a lot cheaper and saves the vanilla plants, [7] since the demands for the world's most popular spice is much below the available supply [8]. The same compound can actually be synthesized out of castoreum from the castor sacs of beavers [9] and be labeled as "natural"[1]. Fortunately, this method is also quite inefficient and unethical.

People should not be afraid of artificial additives because they are in fact the same exact compound as their natural counterparts, only different starting materials, and processing methods. Synthetic additives tend to be purer (have a higher concentration of a certain compound) than natural ones, meaning that it will be more economical for both the producers and consumers. In food industry labs, an array of sensory evaluation methods are used, and it includes professionally trained panelists are used to elaborate the sensory qualities of the food, such as a duo-trio testing method for it to match the standard put as a reference (in this case, the compound's original source)[10].

Synthetic compounds can also be beneficial for people because some foods contain antinutrients[11], an example would be the avidin contained in raw eggs that blocks almost all the absorption rate (bioavailability) of vitamin B7 (biotin)[12], and phytic acids in grains that block the absorption of some minerals (especially zinc) [12]which is the reason for the trend of sprouted grains in recent foods because they can break down the phytic acid so that the body can absorb those nutrients[13].

Allergies may also be a problem from natural ingredients because the compound used for a certain purpose may be derived from a source that people may be allergic to. Several food proteins, including cow's milk, soy proteins, gluten, egg proteins, and peanut proteins cause severe allergic reactions in children and adults[12].Emulsifiers are chemicals that make emulsions happen. Nature uses proteins and phospholipids, and many emulsifiers used in modern food production are based on these natural substances[4].

An example would be how lecithin, an emulsifier derived from soy/eggs may cause allergies to people with soy allergies(though possibilities are low \& usually used in moderation), also, if an animal-based protein emulsifier is added to the food, it would mean that vegans would not be able to consume it. So, safer, non-protein, semi-synthetic alternatives like E325, E327, etc (compounds can be found in nature but almost always synthetically produced) may be used[14]. 
Synthetic materials are also important to prevent foodborne illnesses, like nitrates/nitrites to inhibit the formation of Clostridium botulinum endospores and potentially cause botulism to the consumer. A problem with nitrates and nitrites is their carcinogenicity, however food chemists will always take responsibility and add antioxidants like vitamin $\mathrm{C} / \mathrm{E}$ to balance it out[10].

People should not have fear of what food additives can be consumed or not, because there is a term that a food industry has to follow before adding substances called as the "GRAS list" which is a list of compounds exempt from the food additive definition because they are generally recognized as safe based on "a reasonable certainty of no harm from a product under the intended conditions of use"[10], and it is obligatory for them to follow the "Delaney's Clause" which states "no additive shall be deemed to be safe if it is found to induce cancer when ingested by man or animal[10]."As for the people who are scared of "overdosing" on chemicals, there is a reference called as a "DRI" (Daily Reference Intake) for food technologists to follow to know how much a certain compound is added(applies to both natural and synthetic ingredients) and the nutritional facts label on the food product itself to inform consumers about the serving size of that food. If the rules are followed by both producer and consumer, it will fully be safe.

There are a few drawbacks from artificial chemicals which include the flavor and environmental issues. In the synthesis of artificial flavors/fragrances, only 1 compound is created, whereas, in natural sources, there are a lot of other compounds that can subtly change the sensory attributes of the food. From an environmental aspect, a disadvantage is that most of the compounds are made from organic compounds like petroleum derivatives, and their production may contribute to industrial waste, causing wastewater pollution[15]. However, I think this is still a better alternative in comparison to how natural additives require killing a lot of plants and/animals because this method can be inefficient and unsustainable due to the low availability $\&$ high demand of the required ingredient(s). Below shows a general graph on the dietary guidelines of food components which can be helpful for both producers and consumers:

\subsection{Headings, tables and figures}

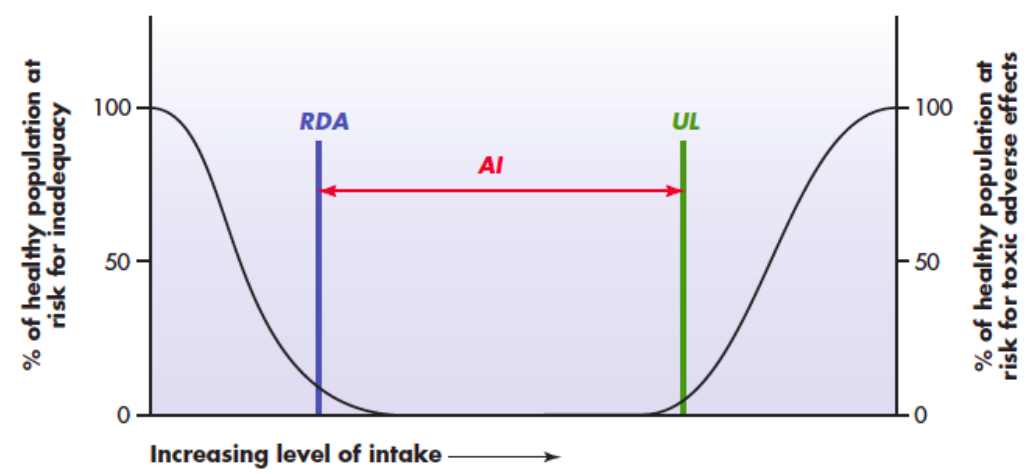

Recommended Dietary Allowance (RDA): The dietary intake level that is sufficient to meet the nutrient requirement of nearly all (97\% to $98 \%$ ) healthy individuals in a particular life stage and gender group. When set for a nutrient, aim for this intake.

Adequate Intake (AI): A recommended intake value based on observed or experimentally determined approximations or estimates of nutrient intake by a group (or groups) of healthy people that is assumed to be adequate - used when an RDA cannot be determined. When set for a nutrient, aim for this intake.

Tolerable Upper Intake Level (Upper Level or UL): The highest level of nutrient intake that is likely to pose no risk of adverse health effects for almost all individuals in the general population. As intake increases above the Upper Level, the risk of adverse effects increases.

Fig. 1. DRI 
Recommended Dietary Allowance (RDA): Recommended Dietary Allowance (RDA): The dietary intake level that is sufficient to meet the nutrient requirement of nearly all (97\% to $98 \%$ ) healthy individuals in particular life stage and gender group. When set for a nutrient, aim for this intake.

Adequate Intake (AI): A recommended intake value based on observed or experimentally determined approximations or estimates of nutrient intake by a group (or groups) of healthy people that are assumed to be adequate - used when an RDA cannot be determined. When set for a nutrient, aim for this intake.

Tolerable Upper Intake Level (Upper Level or UL): The highest level of nutrient intake that is likely to pose no risk of adverse health effects for almost all individuals in the general population. As intake increases above the Upper Level, the risk of adverse effects increases

\subsection{Acknowledgements}

I would like to thank Mrs. Mirza RizqiZulkarnain from IULI for educating me well about food chemistry, and Mr.Hery\& John T.P from UPH for informing me regarding this event, and to the reader of this research paper for your time and scrutiny in reading, I hope this research has educated you in some way.

\section{References}

[1] International Food Information Council (IFIC) and U.S. Food and Drug Administration (FDA), "Overview of Food Ingredients, Additives \& Colors," US Food and Drug Administration. [Online]. Available: https://www.fda.gov/food/foodingredients-packaging/overview-food-ingredients-additives-colors.

[2] A. Kasprak, "Is This Really How Margarine Is Made?," Snopes. [Online]. Available: https://www.snopes.com/news/2018/08/02/how-margarine-is-made/.

[3] Science Learn, "Chemicals everywhere," Science Learn. [Online]. Available: https://www.sciencelearn.org.nz/resources/363-chemicals-everywhere.

[4] Food Additives And Ingredients Association, "What are food additives?," Food Additives And Ingredients Association. [Online]. Available: https://www.faia.org.uk/food-additives/.

[5] Wikipedia, "Vanillin," Wikipedia. [Online]. Available: https://en.wikipedia.org/wiki/Vanillin.

[6] M. M. Bomgardner, "The problem with vanilla," Chem. Eng. News, vol. 94, no. 36, pp. 38-42, 2016.

[7] E. Levine, "Junk Food Costs Less Than Fruits and Vegetables: Are We Surprised?," Serious Eat. [Online]. Available: https://www.seriouseats.com/2007/12/junk-foodcosts-less-than-fruits-and-vegetabl.html.

[8] The Economist explains, "Why there is a worldwide shortage of vanilla," The Economist explains. [Online]. Available: https://www.economist.com/the-economistexplains/2018/03/28/why-there-is-a-worldwide-shortage-of-vanilla.

[9] M. B. Indelicato, "Beaver Butts Emit Goo Used for Vanilla Flavoring," National Geographic. [Online]. Available: https://www.nationalgeographic.com/news/2013/10/beaver-butt-goo-vanillaflavoring/.

[10] A. C. Brown, Understanding food: principles and preparation. Boston, MA: Cengage learning, 2018.

[11] Wikipedia, "Antinutrient," Wikipedia. [Online]. Available: 
https://en.wikipedia.org/wiki/Antinutrient.

[12] S. Damodaran and K. L. Parkin, Fennema's Food Chemistry. Florida: CRC Press.

[13] B. Olson and R. Davis, "A Closer Look At The Sprouted Grains Phenomenon," WisContext. [Online]. Available: https://www.wiscontext.org/closer-look-sproutedgrains-phenomenon.

[14] Federation Of European Specialty Food Ingredients Industries, "Emulsifying Salts," Federation Of European Specialty Food Ingredients Industries. [Online]. Available: https://www.specialtyfoodingredients.eu/ingredients-and-benefits/group/emulsifyingsalts.

[15] Wikipedia, "Environmental impact of the petroleum industry," Wikipedia. [Online]. Available:

https://en.wikipedia.org/wiki/Environmental_impact_of_the_petroleum_industry.

[16] G. Wardlaw, Contemporary nutrition: A functional approach. Pennsylvania: McGraw-Hill Higher Education, 2014. 